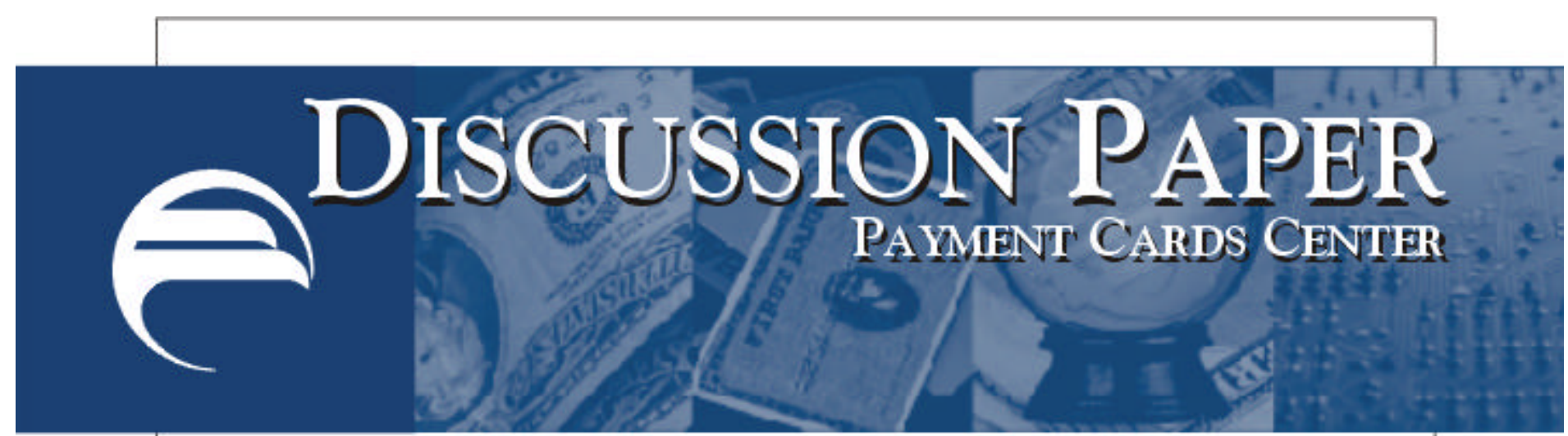

\title{
Managing Consumer Credit Risk
}

\author{
Peter Burns \\ Anne Stanley
}

September 2001

Summary: On July 31, 2001, the Payment Cards Center of the Federal Reserve Bank of Philadelphia hosted a workshop that examined current credit risk management practices in the consumer credit industry. The session was led by Jeffrey Bower, senior manager in KPMG Consulting's financial services practice. Bower discussed "best practices" in the credit risk management field, including credit scoring, loss forecasting, and portfolio management. In addition, he provided an overview of developing new methodologies used by today's risk management professionals in underwriting consumer risk. This paper summarizes key elements of Bower's presentation.

*The views expressed here are not necessarily those of this Reserve Bank or of the Federal Reserve System.

\section{Federal Reserve Bank of Philadelphia}




\section{PORTFOLIO MANAGEMENT AND ANALYTICS}

In Bower's view, consumer credit risk must be understood in terms of a portfolio management strategy that balances capital preservation with capital optimization, that is, “. . a continuous process of identifying and capitalizing upon appropriate opportunities while avoiding inappropriate exposure in such a way as to maximize the value of enterprise." Capturing data across all steps in the customer relationship and integrating information management are the keys to effective portfolio management. While this is a fairly straightforward prescription, executing it is often beyond the scope of many lenders, with the credit card companies generally in the vanguard. Often, the process steps are managed on separate legacy systems, which complicates efforts to integrate information. KPMG consultants find that many firms typically purge specific files before the information is extracted and combined with other data to provide effective portfolio management. The loss of application data, for example, would mean that critical score-card and demographic information would not be available to model behavior in defined customer or risk segments.

\section{BEST PRACTICES IN CREDIT-RISK-MANAGEMENT}

Credit-risk-management practices vary considerably among firms and between segments of the consumer lending industry. To illustrate the variability, Bower described the range of management practices in:

credit decision-making

a credit-scoring

- loss forecasting

$\checkmark$ portfolio management. 
On the front end of the credit process, industry leaders are investing in analytics to improve the credit decision-making process. Building on experience with creditscoring technologies, these leaders are employing expert systems that can adopt to changes in the economy or within specific customer segments.

The use of credit-scoring varies from those that are using only credit bureau data, to those that blend bureau data with other information based on the firm's own experience, to the most advanced applications using adaptive algorithms. These models, used by some of the leading credit card issuers, are updated regularly to reflect changing characteristics of the applicant population. A significant challenge for even the most sophisticated lenders is how to model probable performance when dealing with new customer segments.

Loss forecasting techniques have advanced considerably from their early reliance on historical delinquency rates and charge-off trend analyses. Delinquency flow models and segmented vintage analyses are now commonly used to recognize portfolio dynamics and behavior patterns based on pools with common characteristics. The credit card industry has perhaps gone the furthest with its use of massive segmentation profiles, with the more advanced issuers complementing these profiles with regional economic data and other analytical dynamics.

Over all portfolio management employs all of these techniques with most firms tracking current vs. historical performance and establishing concentration limits for particular risk segments. Some lenders employ risk adjusted return on capital (RAROC) models but Bower and his colleagues argue that multi-year net present value cash flow 
models represent a more effective way to understand optimal risk/reward relationships. In their experience, at this point, only a few firms appear to be testing these more advanced approaches.

Bower concluded this section of the discussion by noting that "the future of consumer credit risk management lies in organizing portfolio performance and account level detail into databases; and then, applying refined analytical models to discern patterns or trends." In doing so, lenders can more effectively manage loss exposures and apply risk-based credit pricing.

\section{ANALYTICAL TECHNIQUES}

Analytical techniques are especially applicable to consumer lending. Consumer portfolios, unlike those in commercial lending, tend to be composed of many relatively homogeneous loans. The relatively common behavior characteristics of portfolio segments make statistical modeling techniques especially useful. As Bower noted, "Analytical techniques that forecast, segment, and classify individual loans into homogeneous pools have provided the competitive advantage to leading-edge consumer lenders.”

The discussion then turned to the application of risk management analytics in dealing with the full spectrum of credit management activities:

response analysis

\ pricing strategies

a loan amount determination

a credit loss forecasting

\ portfolio management strategies 


\section{- collection strategies}

The keys to effective application of analytics across these often interrelated activities are collecting data throughout the business process and managing a common information repository, or risk data warehouse.

In dealing with response analysis, the risk management challenge is to avoid adverse selection consequences that result in increased concentrations of high-risk borrowers. Predictive modeling techniques address this issue but require rigorous response testing to continually improve understanding of customer behavior. Using a range of inputs from origination files (demographics, transactional data, etc.), customer characteristics are segmented and analyzed to develop identifiers of the desired response. Again, the credit card industry is relatively further along in this area, having learned from the painful experiences of a number of issuers in the mid-1990s. In another market, a select group of small-business lenders were also cited as having successfully applied these segmented response analytics to their marketplace.

Pricing strategies for risk remains a challenge for many consumer lenders who tend to "follow the competition." Furthermore, many lenders fail to effectively test pricing models to explore different segments' responses to the trade-offs among annual fees, APR introductory periods, and other pricing variables. Industry leaders use profitability and cash flow modeling to provide insights into portfolio segments and better manage mispriced risk segments. 
Determining the appropriate loan amount directly affects portfolio loss. Judgmental criteria - or, worse, marketing-driven strategies - will generally lead to increased credit exposure. Again, analytical techniques such as cash flow modeling can create outcome scenarios comparing loan amounts relative to risk segments. Statistical methodologies exist that add better control over loan or line setting by determining optimal segments to minimize losses and quantifying probabilities of use.

As we have seen in a number of consumer lending businesses over the years, credit loss forecasts or assumptions used to set pricing and loan amount may well prove inaccurate with the passage of time. Credit card lenders found that historical assumptions for bankruptcy trends proved inadequate during the mid-1990s. In response, most of the industry leaders have greatly enhanced their analytical techniques in this area to better capture portfolio dynamics. Decompositional roll rate modeling, trend and seasonal indexing, and vintage curve techniques to better estimate behavior within individual portfolio cohorts are some of the advanced statistical methodologies currently employed among industry leaders.

Portfolio management is a key issue for consumer lenders as they examine repricing practices and retention strategies and deal with credit line management. Repricing portfolio segments based on judgmental criteria, for example, can lead to lower revenues or increased portfolio risk. Industry leaders are integrating behavioral elements with cash flow profitability modeling to more accurately determine the impact of pricing adjustments on specific customer segments.

Industry leaders understand that collection strategies can have a significant impact on lessening credit losses. In Bower's experience, collection efforts that have 
been augmented with statistical behavior models are demonstrably more effective than those with no behavioral modeling support. He also noted that well-conceived segmentation schemes are leading to targeted collection strategies, decreasing roll rates from one state of delinquency to the next.

\section{SUMMARY}

The tools for improving management of consumer credit risk have advanced considerably in recent years as industry leaders and their advisors have focused on the development of increasingly sophisticated analytical tools. Advances in data warehousing technology and overall computational efficiencies have greatly facilitated these developments. At the same time, application of these new methodologies varies substantially among firms and between industry segments. Generally speaking, the credit card industry tends to be the furthest along the development path, but even - here, variability exists. A number of lending firms have developed highly refined portfolio segmentation designs and enhanced risk-based score-card schemes, but only a few have reached the level of fully integrated models that employ multi-variable regression analysis. At the same time, Bower concluded by noting that risk management practices in the consumer lending business are generally much stronger than in the early 1990s and the industry is far better positioned to weather the current economic downturn than it was a decade ago. 